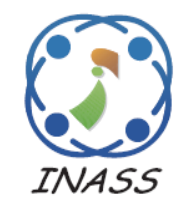

\title{
Improved PTS Technique Based on Sub-Block Weighting Method of PAPR Reduction in OFDM Signals
}

\author{
Kampa Kanthi Kumar ${ }^{1 *} \quad$ Adimulam Yesu Babu $^{2} \quad$ Battula Tirumala Krishna $^{2}$ \\ ${ }^{1}$ Jawaharlal Nehru Technological University, India \\ ${ }^{2}$ Sir CR Reddy college of Engineering, India \\ * Corresponding author's Email: kanthiphd2017@ gmail.com
}

\begin{abstract}
The techniques to achieve Peak-to-Average Power Ratio (PAPR) minimization in Orthogonal Frequency Division Multiplexing (OFDM) signals, has brought more interest among the researchers in recent days. Among various methods for PAPR reduction, the Partial Transmit Sequence (PTS) and Selected Mapping (SLM) has derived successful reduction and improvement compared to other methods. However, it requires a large complexity for obtaining a kind of good performance in PAPR minimization. Thus, it is continuously being revised and improved by the researchers to provide better results. This paper focus on the same motivation mentioned above. It includes an improved PTS with the help of new Sub-block Weighting (SW) method. The improved PTS is combined with SLM technique for diminishing the computational intricacy for OFDM system. Simulation and experimental outcome confirms that the proposed method shows a superior outcome in terms of Complementary Cumulative Distribution Function (CCDF) compared to previous schemes.
\end{abstract}

Keywords: Orthogonal frequency division multiplexing, Partial transmit sequence, Peak-to-Average power ratio, Selected mapping.

\section{Introduction}

OFDM is an efficient technique used for high bit rate transmission systems like digital audio, video, IEEE 802.16 (WiMAX), IEEE 802.11 (WLAN) and so on. OFDM signal's spectral efficiency is increased significantly by separating the bandwidth into subchannels [1-2]. It is appropriate for all types of wireless data broadcast, because of its significance towards frequency selective fading channel. However, it experience issue to transfer the signal due to the problem of high PAPR and causes nonlinear distortion. The regular amplifier with a nonlinear part would give rise to a severe in-band distortion and an undesired out-of-band power of the signal [3]. PAPR is the major problem that affects the performance of OFDM signal transmission by inducing the signal distortion and Bit Error Rate (BER) [4].

Numerous PAPR reduction methods have been proposed such as Interleaving, Window Shaping,
Active Constellation Extension, Companding, Coding, Phase Optimization, SLM and PTS etc. Among those PTS is an effective scheme that achieves high PAPR reduction rate [5-6]. In the PTS method, each block of data input to the Inverse Discrete Fourier Transform (IDFT) is divided into a disjoint sub-blocks, in which each sub-block present in the signal after padding the zero detects an independent IDFT with full size [7]. Outputs from IDFTs are combined properly to achieve minimum PAPR in a significant manner [8]. PTS can able to attain significant performance, but implementation problems arise from a relatively high complexity for searching optimal sequence of phase weight factors. These factors are very effective and have a flexible performance of PAPR reduction. Similarly, SLM gives an effective outcome in OFDM sequence with less PAPR, but it requires Side Information (SI) of phase vectors. Though, the PTS and SLM methods are deliberated to attain satisfactory PAPR reduction performance [9-10]. 
In this scenario, an improved method of SW based SLM-PTS technique that involve in reduction of PAPR on the basis of complexity and also for generating an automatic side information. To increase the OFDM sequences, phase weighting sequences are generated for weighting the first two sub-block sequences, which equivalently makes better reduction of PAPR. Accuracy and the PAPR reduction is maximum in this proposed system.

This paper is composed as follows. In Section II, survey several distinctive PAPR diminishment strategies. In section III, the standards of PTS and SLM strategies are portrayed, to seek the better blend of the phase factors. In Section IV, the execution of Sub-block weighting (SW) based SLM-PTS algorithm for PAPR minimization is assessed by simulation. Conclusion is made in Section V.

\section{Literature review}

Sheng et al. [11] have presented a new PTS scheme named as Space-Frequency Block Coding (SFBC) in MIMO-OFDM systems for minimizing PAPR. Respective, PTS scheme utilized the sample list of powers in sub-blocks in order to generate cost functions for the selection of samples for analysing the peak power of each and every candidate signal, which resulted in the reduction of complexity. Simulation result confirmed that, this particular PTS scheme attained a reduced PAPR and BER rate, with low computational cost. It showed that the PAPR diminishment couldn't moderate the non-linearity of SFBC adequately.

Li et al. [12] have proposed a scheme of partition optimization in LDPC-Coded with PTS for PAPR minimization. In this paper, an optimization problem was also formulated to enhance the enactment of joint decoding by implying optimization in partition. Further, two greedy-based algorithms were used along with this method to solve all major problems. Simulation outcome proved that the joint decoding method along (or) in combination with the proposed partition algorithms delivered a satisfactory errorcorrecting enactment for superior number of PTS groups than the pseudo-random partition method. With the improved performance, better PAPR performance is supported in two ways 1) It provides nearly perfect error-correcting enactment for a superior number of PTS groups, and 2) It possesses faster decoding convergence and lower decoder complexity. While implementing numerous algorithms, the execution time is very high.

Renze et al. [13] have proposed an effective PTS based scheme named Greedy and Genetic Algorithm for OFDM systems. Here, it combined the both traditional PTS method and super-imposed sequence method to attain furthermore PAPR minimization. Training sequences take place in this method has an effective auto-correlation and relative lower PAPR; therefore it can be utilized to achieve further PAPR minimization. Theory and simulations utilized in this system and their result proved that the proposed scheme attained significant PAPR minimization and superiorly decrease the complexity. Though, the number of sub-carriers at the transmitter end is comparably high and it gradually increased the computational complexity.

Ling-Yin et al. [14] have proposed the method of selective weighting, with the help of PTS technique for the reduction of PAPR in OFDM signals. In selective weighting-PTS technique, selective weighting is initially employed for the purpose of diminishing complexity. Unlike Original-PTS method some specialized phase weight sequences has been generated for weighting the first sub-block sequence to enhance the PAPR minimization and its enactment. With a massive computer simulations. This selective weighting-PTS reduced computational complexity greatly and achieves identical PAPR minimization associated to O-PTS. This selective weighting-PTS contained more number of iterations, so that the computational time was quite high.

To overcome the above mentioned drawbacks, an effective weighting algorithm is implemented in PTS, which enhances the procedure acclimated in our anticipated strategy.

\section{Proposed system}

An improved PTS with Sub-Block Weighting (SW) method is combined with SLM technique, to improve the enactment of PAPR with limited complexity. The block diagram of the following experiment is presented in below figure 1 . 


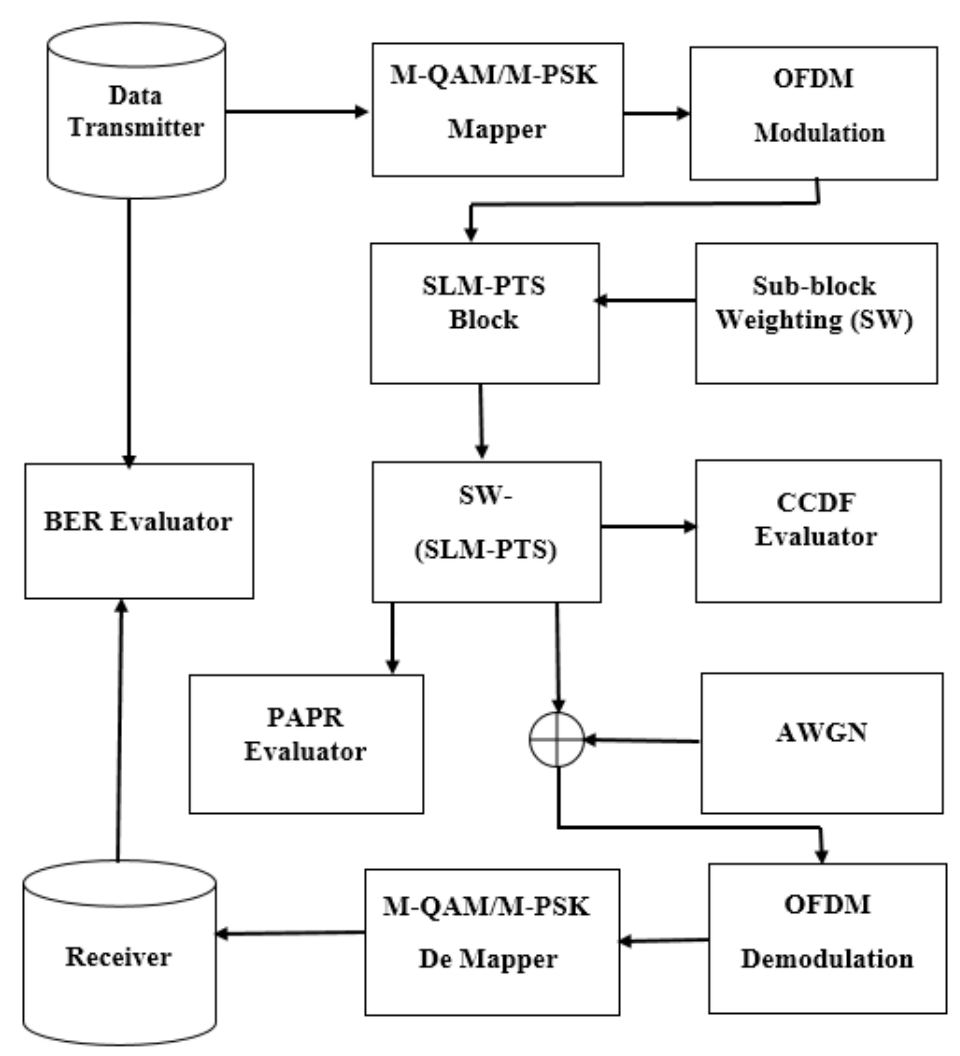

Figure.1 Block diagram of proposed method

This section describes the procedure of OFDM under which PTS method is used for the reduction of PAPR. OFDM system which divides the single radio signal into equally spaced sub-signals to provide less signal distortion rate at high speed broadcast in wireless communication system.

Mathematically, the input frequency domain sequences are denoted as,

$$
X=\left[X_{0}, X_{1}, \ldots . X_{N-1}\right]
$$

Where, $N$ is the dimension of data, $X$ is the input data.

At first, the regulated input frequency samples of OFDM system are modulated into time domain sequences, because the OFDM model encodes only digital dates on multi-carrier frequency. The following sub-carrier sequences are chosen orthogonally to provide multiple transmissions. Modulated time domain signals are represented as follows,

$$
x_{n}=\frac{1}{\sqrt{N}} \sum_{k=0}^{n-1} X_{n} W_{N}^{k n}
$$

Where, $x_{n}$ is the signal further processed by IFFT, $k n$ represents the orthogonal value of the sub-carriers.
To diminish the PAPR in the OFDM sequence of signal, the shifting sequence of data is added in between the data signal.

Summation of the OFDM symbols in the subcarriers are being compared with an individual carrier system, from which the synthesis may lead to high PAPR. This high power is represented as the ratio between maximum power and its average power. It is expressed as,

$$
P A P R=10 \log _{10} \frac{\max \left\{\left|x_{n}\right|^{2}\right\}}{\mathrm{E}\left\{\left|S_{n}\right|^{2}\right\}}
$$

Where, $\mathrm{E}\left\{\left|S_{n}\right|^{2}\right\}$ is specified as the average power of $x_{n}$ and it can be computed as a frequency domain of IFFT unitary transformation type.

SLM is performed from continuous time signal $x_{n}$ by generating the discrete time signal $x_{t}$ by sampling. If sampling at critical sampling or Nyquist rate, $x_{t}$ might have an overly optimistic PAPR value, because it might lose some information at the peak of $X(t)$. Hence, $x_{t}$ is over-sampled by a factor $L$ to provide better estimation result of PAPR value from the continuous time signal $x_{n}$. Over-sampling factor $L$ can be recognized by inserting $(L-1) N$ zeros in the centre of $\mathrm{N}$-point frequency-domain signals $X$ and passing the new LN-point data sequence through 
the LN-point IFFT unit. Therefore, the over-sampled IFFT output from the applied SLM can be expressed as,

$$
x_{t}=\frac{1}{\sqrt{L N}} \sum_{k=0}^{L n-1} \bar{X}_{n} W_{N}^{k l n}, 0 \leq n \leq L n-1
$$

Where $\bar{X}_{n}$ represents the modified input signal. It is shown $\mathrm{L}=4$ is sufficient to capture the peak information of $x_{n}$.

The SLM-PTS technique is included in the OFDM signal formed in order to reduce the PAPR. To precede this, the input block containing data in $X$ is divided by means of a certain partitioning scheme into $M$ disjoint sub-blocks, which are denoted by the representation of vectors $X_{m}, m=$ $1,2, \ldots, M$. Therefore, the acquired equation is mentioned as given in Eq. (5)

$$
X=\sum_{m=1}^{M} X_{m}
$$

Where all the subcarrier positions that present in another block must be zero so those sums of all the sub-blocks provide the original signal.

Addition of disjoint sub-blocks and zeros insert sequences are represented as follows,

Table 1. PTS insertion

\begin{tabular}{|c|c|c|c|c|c|c|}
\hline \multicolumn{7}{|c|}{ X:IFFT Input } \\
\hline \multicolumn{4}{|c|}{ Sub-Blocks (M) } & \multicolumn{3}{|c|}{ Insert sequence (N) } \\
\hline 1 & 2 & $\ldots$ & M-1 & 1 & $\ldots$ & $\mathrm{N}$ \\
\hline
\end{tabular}

\subsection{Sub-block weighting (SW)-SLM-PTS}

The sub-block sequences are independently weighted by Phase Weighting Factors (PWFs). All the weighted sub-block sequences are added to achieve an OFDM candidate sequence, expressed by

$$
\begin{aligned}
& x^{\prime}=\operatorname{IIFT}\left\{\sum_{m=1}^{M} b_{m} X_{m}\right\} ; \\
& x^{\prime}=\sum_{m=1}^{M} b_{m} \operatorname{IFFT}\left\{X_{m}\right\}=\sum_{m=1}^{M} b_{m} X_{m}
\end{aligned}
$$

Where $x_{m}=\operatorname{IFFT}\left\{X_{m}\right\}, b_{m}$ is PWFs for the $m$ sub-block sequence.

Number of OFDM sequences are achieved by implementing various PWFs and the one with lowest PAPR is found for transmitting. Moreover, the side information is required to help the receiver recover the input data correctly. In the proposed SW-(SLMPTS), the SW is initially adopted for simplifying phase weighting procedure. Here, the sub-block sequences are weighted by the allowed PWFs.

Now, all the sub-block sequences are categorized into two parts as original part and weighted part. It is known that without any performance loss, the PWF for the first sub block sequence is one. Hence, for all the sub block sequences, the first half can be viewed as the original part and the weighted part consists of the rest sub blocks. For example, when the number of sub-block sequences is four (i.e. $x_{1}, x_{2}, x_{3}, x_{4}$ ), the two sections can be given as follows.

Original part: $x_{1}, x_{2}$

Weighted part: $x_{3}, x_{4}$

For the weighted part, as $\{1,-1\},\{j,-j\}$ the subblock sequences need to be weighted by PWFs, the corresponding PWF should be generated. Here, two different sets of PWFs are adopted, let $h_{i}, i=$ $1,2, \ldots M$ and $k_{l}, l=1,2, \ldots M$ be the sub-candidate sequences generated by using two different sets of PWFs, where $\mathrm{M}$ and $\mathrm{P}$ represent the number of subcandidate sequences. Based on the linearity of IFFT, the sub-candidate sequences $h_{i}$ and $k_{l}$ can be directly used for obtaining an additional sub-candidate sequence without any phase weighting procedure, given by

$$
y_{i, l}=\frac{\sqrt{2}}{2}\left(h_{i}+k_{l}\right)(i=1,2, . . M ; l=1,2 \ldots P)(7)
$$

Where $y_{i, l}$ denotes a sub-candidate sequence generated by the combination of $h_{i}$ and $k_{l}$.

If PWFs are equivalently incorporated, the factors should be come from the set of PWFs $\{1+j, 1-$ $j,-1+j,-1-j\}$. However, because the factors for weighting the sub-block sequences have the non-unit magnitude, $\frac{\sqrt{2}}{2}$ must be multiplied to revise the magnitude of PWF and obtain the unit magnitude, shown in Eq. (7)

Hence, we can achieve the sub-candidate sequences of these two parts as follows.

$$
\begin{aligned}
& \text { Original part: } y^{\prime}=x_{1}+x_{2} \\
& \text { Weighted part: } h_{1}=x_{3}+x_{4} \text {, } \\
& h_{2}=x_{3}-x_{4} \text {, } \\
& h_{3}=-x_{3}+x_{4} \text {, } \\
& h_{4}=-x_{3}-x_{4} \text {, } \\
& y_{i, l}=\frac{\sqrt{2}\left(h_{i}+k_{l}\right)}{2} i, \quad l=1,2,3,4
\end{aligned}
$$

By combining the sub-candidate sequences from these two parts, all the OFDM candidate sequences are demonstrated as follows,

$$
\left\{\begin{array}{c}
y^{\prime}+h_{i}, i=1,2,3,4 \\
y^{\prime}+k_{i}, i=1,2,3,4 \\
y^{\prime}+y_{i, l}, i, l=1,2,3,4
\end{array}\right\}
$$


Thus, when the number of sub-block sequences is four, we can attain twenty-four OFDM candidate sequences at most.

\subsection{Computational complexity}

In proposed SW-(SLM-PTS) scheme, the computational complexity is reduced by generating the additional sub-candidate sequences in the weighted part. To show the advantage of proposed system in complexity reduction against Conventional-(SLM-PTS), Computational Complexity Reduction Ratio (CCRR) is calculated, which is defined by Eq. (10).

$$
C C R R=\left(1-\frac{\text { complexity of } S W-(S L M-P T S)}{\text { complexity of } C-(S L M-P T S)}\right)
$$

Table 2. Performance Evaluation in terms of CCRR

\begin{tabular}{|c|c|c|c|c|}
\hline $\begin{array}{c}\text { Complex } \\
\text { Computatio } \\
\text { n }\end{array}$ & $\begin{array}{c}\text { No of } \\
\text { Sub- } \\
\text { block } \\
\text { s } \\
\text { (M) }\end{array}$ & PTS & $\begin{array}{c}\text { Propose } \\
\mathbf{d}\end{array}$ & $\begin{array}{c}\text { CCR } \\
\mathbf{R} \\
(\boldsymbol{\%})\end{array}$ \\
\hline $\begin{array}{c}\text { Multiplicatio } \\
\text { n }\end{array}$ & 4 & $\begin{array}{c}2048 \\
0\end{array}$ & 10240 & $50 \%$ \\
\hline
\end{tabular}

Table 3. Simulation parameters

\begin{tabular}{|c|c|}
\hline Simulation Parameters & Type / Values \\
\hline $\begin{array}{c}\text { Number of random OFDM } \\
\text { blocks (X) }\end{array}$ & 10000 \\
\hline Number of sub-carriers & 512,1024 \\
\hline Number of sub-blocks (M) & 4 \\
\hline Over-sampling factor(L) & 4,8 \\
\hline Modulation scheme & QPSK \\
\hline $\begin{array}{c}\text { Optimal Phase weighting } \\
\text { factor (b) }\end{array}$ & $1,-1, \mathrm{j},-\mathrm{j}$ \\
\hline
\end{tabular}

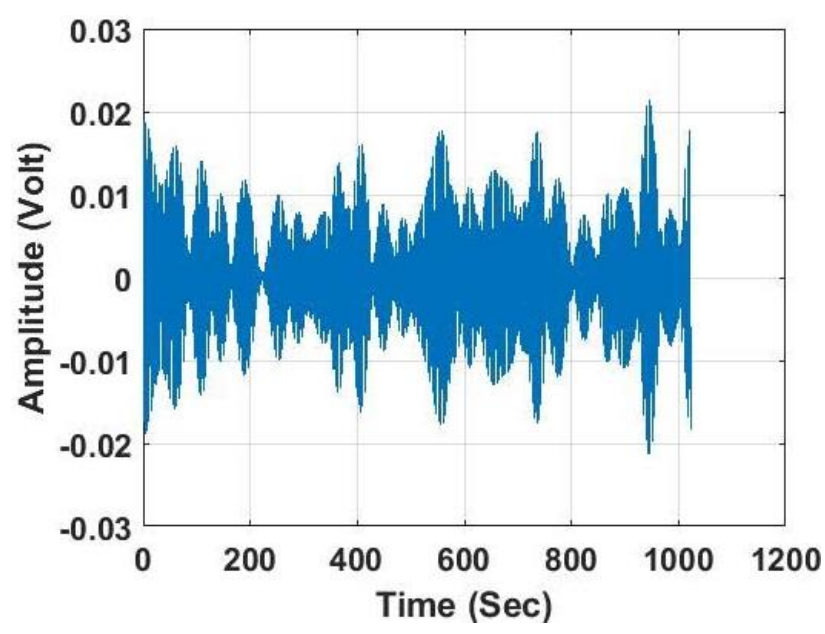

Figure.2 OFDM signal for one symbol period

\section{Results and discussion}

The analysis of the proposed method SW- (SLMPTS) was carried out using MATLAB 8.6.0.267246 (R2015b). In the OFDM system, the proposed technique was applied to the sub-blocks of un-coded information, which was modulated by QPSK modulation, and the phase rotation factors were transmitted directly to receiver through sub-block. Performance estimation was done in terms of CCDF and the simulation parameters considered for this analysis are illustrated in Table 1.

Figure 2 shows the selected period of signal form generated random signal which has 10000 samples of random numbers. We have considered 1000 samples for one symbol period, which is represented in both frequency and time domain and these samples are further processed for DSI, Block portioning, parallel to serial conversion and transmission.

The proposed SW-(SLM-PTS) performance is evaluated by comparing the following parameters 1 . Variation in Number of sample, 2. BER and Signal to Noise Ratio 3.comparision of channel.

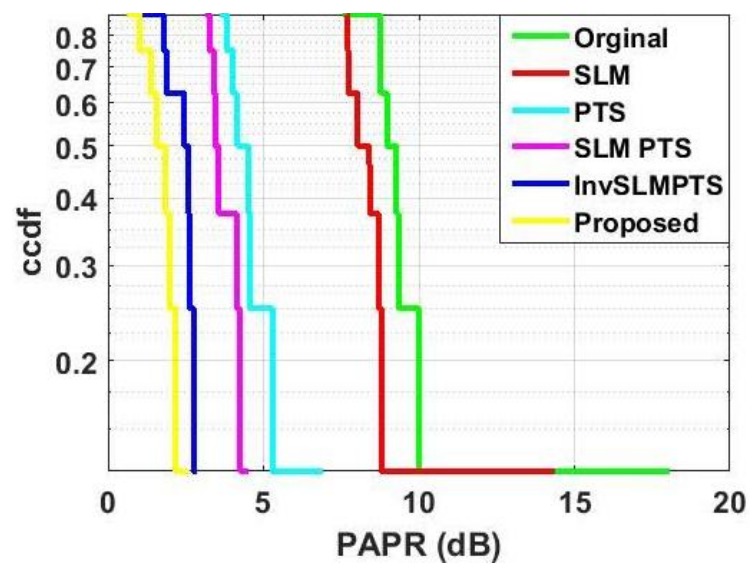

Figure.3 Comparison of existing and proposed system for IFFT length 1024

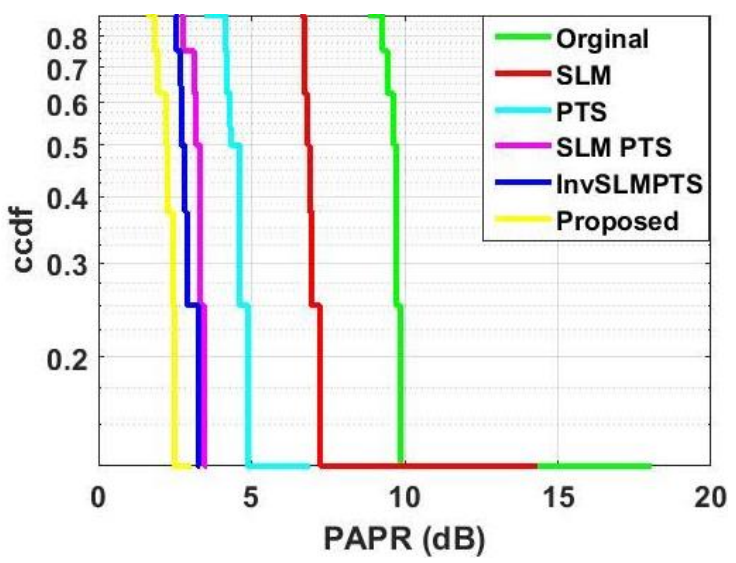

Figure.4 Comparison of existing and proposed system for IFFT length 512 


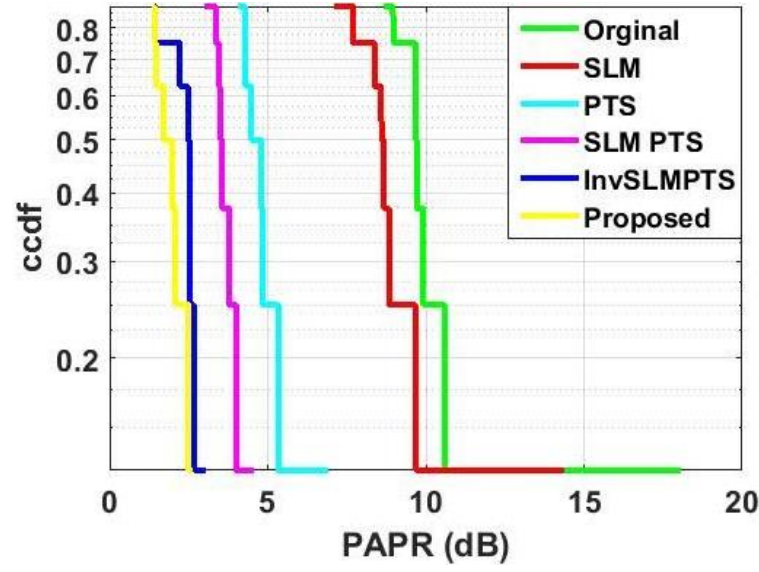

Figure 5. Comparison of existing and proposed system for IFFT length 256

\subsection{Number of samples comparison}

The comparison of the samples are done with the OFDM signals of IFFT length 1024, 512, and 256 respectively. The maximum iteration of SLM and PTS is 4 and 8 . The above scenario is mentioned for the 1024 IFFT symbol. If the IFFT length is changed to different sample size such as 512 and 256 the PAPR hit value also get reduced.

Figure 3 shows the PAPR value of the original OFDM signal is $11.78 \mathrm{~dB}$ and the other approaches like SLM, PTS and proposed method shows $8.15 \mathrm{~dB}$, $4.64 \mathrm{~dB}$ and $2.21 \mathrm{~dB}$ respectively. It confirms that our proposed scheme outperforms the existing schemes. Here, the maximum iteration of SLM and PTS is 4 and 8 .

The above scenario is mentioned for the 1024 IFFT symbol. If the IFFT length is changed to different sample size such as 512 and 256 the PAPR hit value also get reduced. Our proposed method gives $0.32 \mathrm{~dB}$ for 256 IFFT lengths and $0.95 \mathrm{~dB}$ for 512 IFFT length. The comparison of existing systems with the proposed method is proceeded for 512, and 25. This is shown in the below Figs. 4 and 5 .

From the above Figs. 3, 4 and 5, we examined that the proposed method shows better PAPR reduction value than the other existing algorithms. Respective figures clearly describes that the PAPR hit value is diminished after applying reduced IFFT length. The 256 IFFT length gives $2.26 \mathrm{~dB}$ (saturation point at which the PAPR curve meets zero) in PAPR reduction, and this shows the best solution in this analysis. Here, the side information are automatically selected by employing a new weighting scheme. Whereas, the computational complexity is gradually diminished. Experimental outcome of proposed scheme is compared with previous weighting methods.
Boonsrimuang et al [15] illustrated a combined weighting on PTS method, which reduces PAPR up to $8.75 \mathrm{~dB}$. In this study, the sub-block partition is enormous in input data. Due to this procedure the computation complexity is quiet increased. On the other hand, Hameed et al [16] presented a technique named improved weighting factor in PTS method that reduce PAPR up to $6.32 \mathrm{~dB}$. Though, it is a cluster based partition scheme, the input data blocks are partitioned into three different types such as adjacent partition, interval partition, and random partition. While performing with several partition methods, the computational complexity is comparably high. Compared to these two previous weighting schemes, our proposed method works effectively in terms of complexity and PAPR reduction.

\subsection{BER vs. SNR for signal length}

Figure 6 shows the BER vs SNR curves for the proposed system. BER gives the end to end performance of the system during various SNR values. BER is calculated using 20 iterations and with different IFFT sizes of OFDM system. From Fig. 6, we can observe that 1024 IFFT signal length achieves better performance when compared with various IFFT length i.e. Lesser BER for the proposed system. From the result is clear that higher IFFT length produces better BER. The above mentioned scenario is mentioned for the AWGN channel.

Figure 7 specifies the comparison of rician channel and AWGN channel. Due to light weight, AWGN channel shows a superior result in PAPR minimization compared with rician channel.

Figure 8 determines the comparison of proposed (SW-SLM-PTS) and (C-SLM-PTS). It confirms that the IFFT length of 512 and 1024 for proposed shows a significant result in PAPR minimization of $2.62 \mathrm{~dB}$ and $3.47 \mathrm{~dB}$.

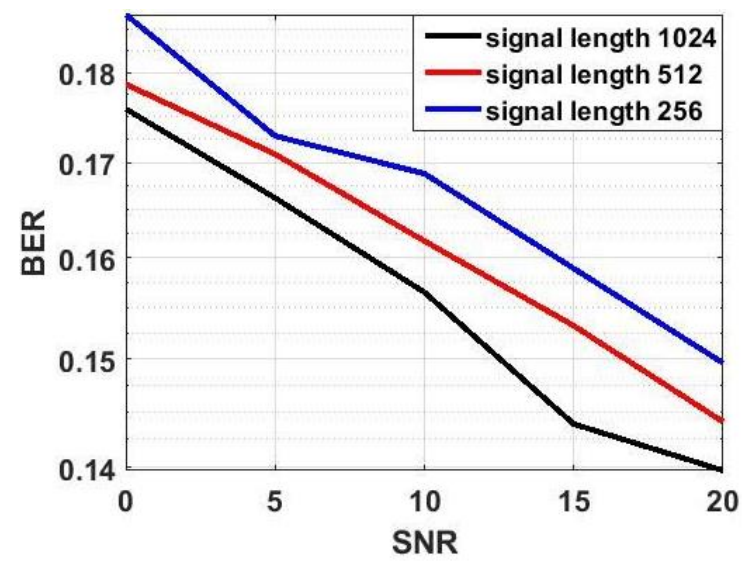

Figure.6 BER Vs SNR comparison for different IFFT length of $1024,512,256$ 


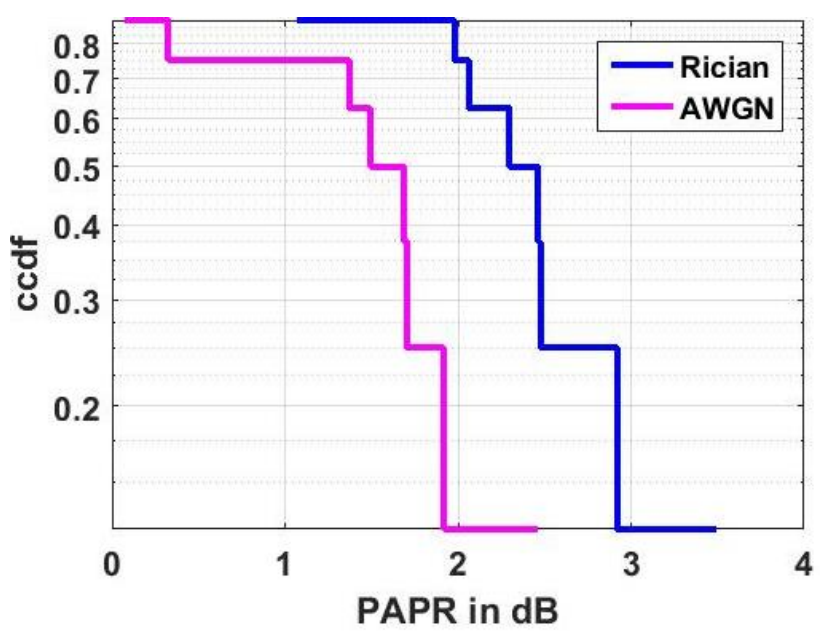

Figure.7 Proposed comparison between Rician and AWGN channel

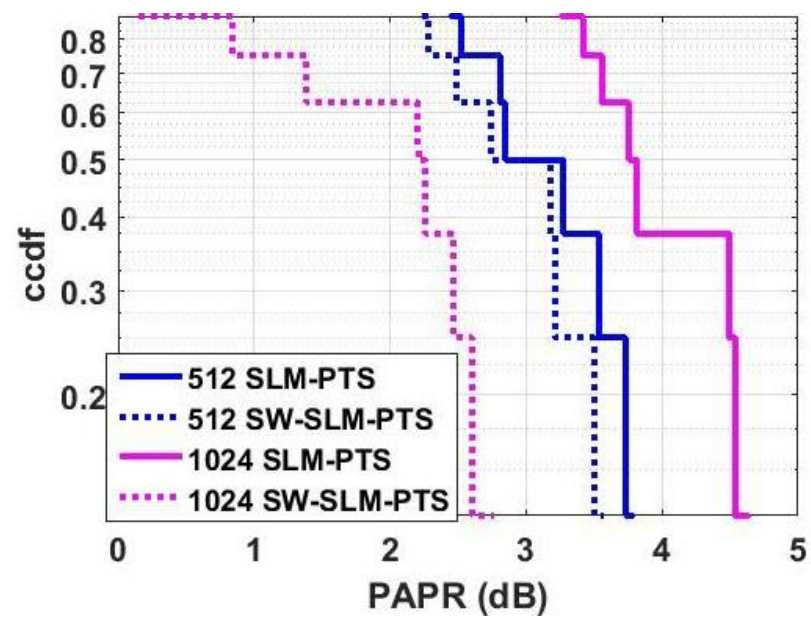

Figure.8 Proposed comparison for different channels with the IFFT length of 512 and 1024

\section{Conclusion}

Generally, the PTS scheme is utilized for minimizing the PAPR in OFDM system. Though, a major concern in PTS is that, it requires numerous reverse fast Fourier or wavelet changes, which attains high computational complexity. To reduce the complexity in PTS, an improved SW scheme was employed with the combination of SLM. This weighting scheme choose the side information automatically. So, the loss of side information is diminished. Hence, the computational complexity in PTS is also gradually decreased. This proposed SW method attempts to provide satisfactory result in any kind of IFFT length. In future, the PAPR in OFDM frameworks is further diminished by employing new approach.

\section{References}

[1] S. Pyla, K.P. Raju, and N. Balasubrahmanyam, "Investigation of Optimum Phase Sequence for Reduction of PAPR Using SLM in OFDM System", In: Proc. of Microelectronics, Electromagnetics and Telecommunications, pp. 255-265, 2016.

[2] C. Duanmu, and H. Chen, "Reduction of the PAPR in OFDM systems by intelligently applying both PTS and SLM algorithms", Wireless personal communications, Vol.74, No.2, pp.849-863, 2014.

[3] J.Y. Woo, H.S. Joo, K.H. Kim, J.S. No, and D.J. Shin, "PAPR analysis of class-III SLM scheme based on variance of correlation of alternative OFDM signal sequences", IEEE Communications Letters, Vol.19, No.6, pp.989992, 2015.

[4] H.G. Ryu, "PAPR reduction using sub block phase weighting (SPW) method in OFDM communication system", In: Proc. International Conf. on Wireless Communications, Networking and Mobile Computing, Vol. 1, pp. 249-252, 2005.

[5] T. Deepa, and R. Kumar, "Performance Evaluation of a Low Complexity Row-Column Transform Approach for SLM Based OFDM Transmission System", Wireless Personal Communications, Vol.87, No.4, pp.1357-1369, 2016.

[6] A.S. Rathor, M. Pathela, and S.S. Rana, "PAPR Reduction in OFDM System using Wavelet SLM and PTS Technique", International Journal of Computer Applications, Vol.98, No.19, 2014.

[7] A. Joshi, K. Nigam, and S. Bansal, "IterativeGrouping and image PTS for PAPR reduction in OFDM system", In Proc. of $3 r d$ International Conf. on Signal Processing and Integrated Networks (SPIN), pp. 195-199, 2016.

[8] L. Yang, R.S. Chen, K.K. Soo, and Y.M. Siu, "An efficient sphere decoding approach for PTS assisted PAPR reduction of OFDM signals", AEU-International Journal of Electronics and Communications, Vol.61, No.10, pp.684-688, 2007.

[9] T. Sravanti, and N. Vasantha, "A combined PTS \& SLM approach with dummy signal insertion for PAPR reduction in OFDM systems", In: Proc. of International Conf. on Computer and Communications Technologies (ICCCT), 2014.

[10] A. Goel, P. Gupta, and M. Agrawal, "Generalized M-2M mapping scheme for SLM and PTS based OFDM systems without side- 
information", Wireless personal communications, Vol.74, No.2, pp.285-305, 2014.

[11] S.J. Ku, "Low-complexity PTS-based schemes for PAPR reduction in SFBC MIMO-OFDM systems", IEEE Transactions on Broadcasting, Vol.60, No.4, pp.650-658, 2014.

[12] L. Li, D. Qu, and T. Jiang, "Partition optimization in LDPC-Coded OFDM systems with PTS PAPR reduction", IEEE Transactions on Vehicular Technology, Vol.63, No.8, pp.4108-4113, 2014.

[13] R. Luo, C. Zhang, N. Niu, and R. Li, “A LowComplexity PTS Based on Greedy and Genetic Algorithm for OFDM Systems", Chinese Journal of Electronics, Vol.24, No.4, pp.857861, 2015.

[14] L.Y. Wang, H. Yuan, and L.G. Liu, "Selective Weighting PTS PAPR Reduction Scheme in OFDM Systems", In: Proc. of Electronics, Communications and Networks, Singapore, pp.343-349, 2016.

[15] P. Boonsrimuang, S. Sanpan, P. Boonsrimuang, T. Paungma, and H. Kobayashi, "Improved PTS Method with New Weighting Factor Technique for OFDM Signal”, In: Proc. Vehicular Technology Conference, pp. 1-5, 2009.

[16] L.S. Hameed, "A combined weighting and PTS technique for PAPR reduction in OFDM signals", In: Proc. 2nd International Conf. on Current Trends in Engineering and Technology (ICCTET), pp. 228-232 2014. 